\title{
FACTORES DE RIESGO ASOCIADOS CON ENFERMEDAD PERIODONTAL EN MUJERES EN ESTADO DE EMBARAZO
}

\author{
Ximena Andrea Cerón-Bastidas ${ }^{*}, \mathrm{MSc}_{1}$, Diana Isabel Josa, Esp. ${ }_{1}$ \\ ${ }_{1}$ Facultad de Odontología, Universidad Cooperativa de Colombia, sede Pasto, Colombia
}

Recibido: 10 de septiembre del 2012. Aprobado: 16 de noviembre del 2012.

*Autor de correspondencia: Ximena Andrea Cerón, Universidad Cooperativa de Colombia, Torobajo, Pasto, Colombia, (57) 273132 00, Calle 18 n. ${ }^{\circ} 47-150$, correo electrónico: ximena.ceron@campusucc.edu.co

Cómo citar este artículo: Cerón-Bastidas XA, Josa DI. Factores de riesgo asociados con enfermedad periodontal en mujeres en estado de embarazo. Revista Nacional de Odontología. 2013; 9(16): 75-81.

Resumen. La enfermedad periodontal ha sido asociada a un gran número de factores de riesgo, y a su vez se puede considerar como un factor de riesgo importante para la evolución de algunos eventos patológicos; tal es el caso de la asociación con parto pre-término, bajo peso al nacer y pre-eclampsia. La enfermedad periodontal es una entidad clínica multifactorial en la que se ven involucrados mecanismos inflamatorios que también están presentes en condiciones adversas del embarazo. La literatura reafirma estos hallazgos y ha orientado las investigaciones hacia las bases celulares y moleculares en busca de una explicación certera que permita crear medidas preventivas de gran impacto. Esta revisión bibliográfica tomó en cuenta información a partir del 2000, incluidos revisiones y estudios experimentales con respecto a los mecanismos celulares que explican las posibles alteraciones en los tejidos periodontales durante el embarazo, que desencadenan consecuencias como parto pre-término, pre-eclampsia, bajo peso al nacer.

Palabras clave: embarazo, factores de riesgo, periodontitis, salud oral.

\section{Risk Factors Associated with Periodontal Disease in Pregnant Women}

Abstract. Periodontal disease has been associated to a vast number of risk factors and may be in turn considered as a risk factor for the development of some pathological events, such as premature birth, low weight at birth and pre-eclampsia. Periodontal disease is a multifactorial clinical entity in which inflammatory mechanisms that are also present in adverse conditions for pregnancy are involved. The literature confirms these findings and has oriented research towards cellular and molecular bases in search of a precise explanation that may allow for preemptive measures with great impact. This bibliographical review considered information starting in the year 2000, including reviews and experimental studies concerning the cellular mechanisms that explain possible alterations in periodontal tissue during pregnancy that may unchain consequences as premature birth, pre-eclampsia and low weight at birth.

Keywords: pregnancy, risk factors, periodontitis, oral health.
Fatores de risco associados com doença periodontal em mulheres grávidas

Resumo. A doença periodontal vem sendo associada a um grande número de fatores de risco e, por sua vez, pode ser considerada como um fator de risco importante para a evolução de alguns eventos patológicos; tal é o caso da associação com parto pré-termo, baixo peso ao nascer e pré-eclâmpsia. A doença periodontal é uma entidade clínica multifatorial na qual se vêm envolvidos mecanismos inflamatórios que também estão presentes em condições adversas da gravidez. A literatura reafirma essas descobertas e tem orientado as pesquisas às bases celulares e moleculares em busca de uma explicação exata que permita criar medidas preventivas de grande impacto. Esta revisão bibliográfica levou em consideração informação a partir de 2000, incluídos revisões e estudos experimentais a respeito dos mecanismos celulares que explicam as possíveis alterações nos tecidos periodontais durante a gravidez, que desencadeiam consequências como parto pré-termo, pré-eclâmpsia, baixo peso ao nascer.

Palavras-chave: gravidez, fatores de risco, periodontite, saúde oral. 


\section{Introducción}

Durante el embarazo, la mujer experimenta grandes cambios tanto extrínsecos como intrínsecos, los cuales están relacionados directamente con el aumento del nivel hormonal, que prepara al cuerpo humano para la creación y desarrollo de una nueva vida dentro del útero de la madre.

La formación de un nuevo ser requiere, entonces, que todas la funciones del cuerpo de la madre y del feto se conjuguen y exista un equilibrio [1]; por otro lado, cuando se interrumpe alguna cadena fisiológica o se presentan alteraciones sistémicas, se pone en peligro la formación, vida y desarrollo del feto [2].

En este artículo se pretende establecer, a través de una revisión bibliográfica exhaustiva, la relación que existe entre factores de riesgo asociados con enfermedad periodontal en mujeres en estado de embarazo.

\section{Cambios endocrinos}

Las principales hormonas durante el embarazo son los estrógenos, que aumentan gradualmente hasta el octavo mes, y la progesterona, la cual mantiene las concentraciones constantes hasta el último mes. Los niveles de estrógenos aumentan en relación con la progesterona en la sangre de 100:1 en el embarazo temprano y 01:01 a término [3].

La placenta adquiere un papel importante en el intercambio materno-infantil, ya que se convierte en un órgano endocrino que tiene la capacidad de producir y permitir el aumento hormonal durante el embarazo. La progesterona y los estrógenos cumplen funciones fundamentales; en la progesterona una de ellas es el mantenimiento de la implantación durante la etapa del embarazo, y en los estrógenos su función principal es ayudar al incremento del flujo de sangre en la placenta garantizando que el feto reciba los nutrientes necesarios. Estas hormonas tienen efectos sobre el sistema vascular, mantienen el endometrio, preparan la glándula mamaria participando en la lactogénesis y modulan el sistema inmune [4].

\section{Respuesta del epitelio y tejido conectivo al ataque bacteriano}

Las respuestas inmune específica y no específica son las implicadas en evitar la propagación de la inflamación en los tejidos; estas reacciones son autoconservadoras. Las células fagocíticas, como son los polimorfonucleares (PMN) leucocitos, macrófagos y linfocitos, son las encargadas de la defensa del huésped. Los cambios vasculares son los primeros eventos que se presentan como respuesta del hospedero frente a la placa bacteriana, por lo cual los vasos sanguíneos de los tejidos periodontales se dilatan y aumentan su permeabilidad; los leucocitos migran de la sangre rápidamente al sitio de la inflamación, el tejido conectivo inflamado empieza a ser infiltrado por células PMN y otras células migratorias, produciendo enzimas proteolíticas, dando como resultado la degradación de la matriz extracelular; los vasos gingivales y los fibroblastos componen el tejido conectivo y son un blanco importante para las hormonas [5].

Por otra parte los linfocitos $\mathrm{T}$ parecen ser más sensibles a los cambios hormonales que los linfocitos $\mathrm{B}$. Las células T-helper, subgrupo de los linfocitos T, son células moduladoras de la respuesta inmune, y se consideran como una importante fuente de citoquinas; estas, a su vez, se dividen en dos grupos, uno de tipo $\mathrm{TH}-1$, que produce una respuesta pro-inflamatoria, $\mathrm{y}$ otro de tipo $\mathrm{TH}-2$, que genera acciones antiinflamatorias, como la interleucina-6 (IL-6). En un embarazo normal la respuesta inmuno-sistémica se desvía hacia la secreción de interleucina 6 , y su producción es modulada por hormonas esteroides, por la progesterona y la testosterona, las cuales disminuyen la producción de L-6 de los fibroblastos gingivales humanos, que a la vez resulta en la baja resistencia a procesos inflamatorios. El aumento de las concentraciones hormonales de estrógenos y progesterona en el embarazo estimula la producción de prostaglandina E2, que puede exacerbar la inflamación gingival $[6,7]$.

\section{Cambios microbiológicos en la flora supra y subgingival}

La flora subgingival puede ser modificada por el embarazo [8]. Estudios longitudinales y transversales demuestran cambios microbianos en cuanto al incremento del número de algunos microorganismos [9], lo cual también sucede cuando existe un cuadro inflamatorio crónico periodontal y el número de bacterias Gram negativas anaerobias se incrementan con respecto a las aerobias. Durante el embarazo, los cambios en la cavidad oral se presentan principalmente en la vasculatura gingival de la encía, entre ellos: coloración rojo oscura, inflamación, consistencia blanda que sangra con facilidad, y algunas veces se pueden desarrollar agrandamientos gingivales localizados $[10,11]$. 
La posibilidad de inflamación gingival durante el embarazo se asocia probablemente con el aumento hormonal en este periodo, el cual se reduce en el parto [12]; el tejido gingival está expuesto por hormonas esteroideas, en las cuales existen receptores especiales que circulan en la sangre, y en menor proporción en la saliva, y pueden alterar la flora subgingival, aumentando el flujo vascular e influir en cambios en el sistema inmunológico y metabólico del tejido conectivo [13].

Son diversas las enfermedades que se han relacionado con la enfermedad periodontal; entre ellas, la pre-eclampsia $[14,15]$, enfermedades cardiovasculares, diabetes mellitus, enfermedades respiratorias, osteoporosis, infecciones y bajo peso al nacer [16]. Varios estudios demuestran que la enfermedad periodontal puede tener una gran influencia en la condición de la salud sistémica del individuo. La periodontitis se origina de una invasión de bacterias Gram negativas sobre los tejidos de protección y soporte del diente, lo cual hace que se produzca una respuesta inmuno-inflamatoria continua que genera daños locales y eventos contraproducentes a nivel sistémico $[17,18]$.

La periodontitis es una inflamación inespecífica, vascularizante y proliferativa, con grandes cantidades de células inflamatorias infiltradas; por ello es importante controlar estos factores que preexisten en el cuadro clínico periodontal [19].

Inpijn y colaboradores llevaron a cabo un estudio sobre la respuesta inmune de la encía en mujeres embarazadas expuestas a una gingivitis experimental, en el que se evidenció un mayor número de células T-helper. Encontraron que existía una mayor inflamación gingival y sangrado al sondaje en la región de los dientes anteriores que en regiones molares y premolares, aunque la cantidad de placa fue mayor en los dientes posteriores. En este estudio también se señaló que los macrófagos tienen funciones múltiples: además de su acción antimicrobiana, desempeñan un papel importante en la remodelación de los tejidos durante la inflamación, están involucrados en la regulación de la proteólisis extracelular y producen el plasminógeno inhibidor del activador del tipo 2 (PAI-2), que realiza la proteólisis de los tejidos [20]. Las mujeres con un cuadro clínico de gingivitis o periodontitis tienen bajas concentraciones de PAI- 2 en el fluido gingival crevicular (GCF), mientras en mujeres con tejidos periodontales sanos tienen altas concentraciones de PAI-2, debido probablemente a que protege el tejido conectivo de la degradación excesiva [21].

Loe y Sillnnes demostraron en su estudio que las mujeres embarazadas tienen mayor prevalencia de gin- givitis en $100 \%$, que aquellas que no se encuentran en este estado, con $30 \%$. La gravedad de los cambios inflamatorios gingivales ha demostrado ser más frecuente durante el embarazo que después del parto, aunque no se produzcan cambios significativos en la cantidad de placa [22]. Los autores en mención determinaron que los primeros signos de inflamación gingival aparecen a partir del segundo hasta el octavo mes, y que el cuadro clínico se acentúa en los meses tercero y octavo, después del cual se produce una mejoría hasta el último mes. Posterior al parto, se halló que el estado gingival era similar desde el primero hasta el segundo mes de embarazo [23.]

Ringsdorf y colaboradores, en otro estudio transversal realizado en 330 mujeres embarazadas, demostraron una correlación positiva entre la movilidad de los dientes durante el embarazo y la gravedad de la gingivitis [24]. Por otra parte, existen estudios en los que no se encontró relación entre enfermedad periodontal y el estado de embarazo [25], como es el realizado por Maier y Orban, referenciado por Merjan, en el que se tomó una muestra de 530 mujeres embarazadas y no se encontró prevalencia ni relación entre gingivitis en mujeres embarazadas [3]. Otros estudios de Tilakaratne y colaboradores y Cohen y colaboradores no encontraron ningún efecto del embarazo relacionado con los niveles de inserción periodontal. La mayoría de los estudios han demostrado que los cambios inflamatorios mencionados se limitan a la encía [3].

Pocos estudios se han publicados sobre el efecto del embarazo en cuanto a los niveles de inserción periodontal, la cual probablemente requiere un estado inflamatorio crónico más extenso que los nueve meses de embarazo [26, 27].

Algunos estudios demuestran que puede existir relación entre enfermedad periodontal con parto prematuro y bajo peso al nacer [28, 29]. La Federación Internacional de Obstetricia y Ginecología (FIGO) y la Organización Mundial de la Salud (oms) denominan parto prematuro o pre-término al que se encuentra entre la semana 22 y antes de la semana 37 de gestación, y el bajo peso al nacer como aquel peso inferior a $2500 \mathrm{~g}$, concepto aprobado por la Asamblea Mundial de la Salud en 1976 [30].

Estudios recientes han demostrado una asociación entre estas condiciones; sin embargo, no es claro si existe o no una relación causal entre ellos, ya que pueden influir varios factores maternos como son cuello uterino corto y la vaginosis bacteriana, que pueden interactuar sinérgicamente con la periodontitis e inducir partos prematuros [31, 32]. 
Un estudio realizado por Calabrese y colaboradores, de casos y controles sobre una población de 110 mujeres tailandesas embarazadas, confirmó que la deficiente salud periodontal es un factor de riesgo potencial independiente para el bajo peso al nacer. Sin embargo, en otra evaluación parece ser que el estado periodontal de estas mujeres se describe sólo a través de un índice de detección parcial, índice de necesidad de tratamiento periodontal de la comunidad (CPITN), y por tanto no estaba suficientemente detallado [8].

Mitchell-Lewis, en una de cohorte con $213 \mathrm{mu}$ jeres, llegó a la conclusión de que la terapia periodontal (IHO, raspaje y pulido) es capaz de producir cerca de $29 \%$ de reducción en PLBW $[33,34]$. No obstante, este estudio se realizó sobre una población de mujeres muy jóvenes (adolescentes, edad media de 16,7 años de edad) procedentes de muy bajo nivel socioeconómico y que se caracterizó por un alto número de abandonos (el 25\% del total de las pacientes) $[35,36]$.

Boggess y colaboradores, en el 2006, analizaron la sangre del cordón umbilical de 640 recién nacidos y midieron la proteína $C$ reactiva, IL- $1 \beta$, TNF- $\alpha$, PGE2, 8 isoprostan y los niveles de IgM contra los patógenos periodontales (Campylobacterrecto, Peptostreptococcusmicros, Prevotellanigrescens, Prevotellaintermedia y Fusobacteriumnucleatum) [30]. El riesgo de prematuridad fue mayor cuando se detectó IgM contra al menos un patógeno periodontal, y aún más cuando los niveles de mediadores inflamatorios altos fueron encontrados; los autores evidencian cómo la exposición fetal a las componentes moleculares de las bacterias periontopatógenas y la reacción inflamatoria pueden inducir a un parto prematuro y a que un bebé presente bajo peso al nacer [37].

El control de la enfermedad periodontal durante el embarazo podría contribuir a minimizar resultados adversos [38]; el efecto de las intervenciones periodontales en estas mujeres fue evaluado en un estudio prospectivo diseñado para examinar la relación entre la enfermedad periodontal y los recién nacidos prematuros con bajo peso al nacer en una cohorte de 164 mujeres jóvenes embarazadas y puérperas, en el que 74 fueron sometidas a profilaxis oral durante el embarazo y 90 no recibieron tratamiento periodontal. El índice de peso bajo de los nacimientos prematuros fue menor entre las mujeres que recibieron tratamiento periodontal en comparación con los que no $(13,5 \%$ vs.18,9\%) [39, 40].

Sant'Ana y colaboradores, en el 2011, sugieren que la realización de un tratamiento periodontal durante el segundo trimestre de la gestación podría disminuir el riesgo de desarrollo de resultados adversos del embarazo, lo que podría implicar que la enfermedad periodontal puede ser considerada como un factor de riesgo especialmente parto pre-término y bajo peso al nacer $[41,42]$.

\section{La proteína $\mathrm{C}$ reactiva y enfermedad periodontal}

La proteína $\mathrm{C}$ reactiva (PCR) es una reactante de fase aguda generada por el hígado en respuesta a la presencia de citoquinas inflamatorias como lainterleuquina6 (IL-6 ), IL-1 y el factor de necrosis tumoral alfa, cuando se presentan estímulos inflamatorios generados por el calor, traumatismos, infecciones e hipoxia. Los niveles circulantes de PCR sirven como un marcador de la inflamación sistémica y se han asociado con enfermedad periodontal $[43,44]$. Puede existir alguna relación entre la PCR y los resultados adversos del embarazo, incluyendo el parto pre-término [45] y la pre-eclampsia [46]. Además, la enfermedad periodontal se ha asociado con un mayor riesgo de parto pre-término y bajo peso al nacer $[47,48]$.

Los recién nacidos pre-término presentan un riesgo elevado de muerte, discapacidades del desarrollo neurológico, deterioro cognitivo y trastornos conductuales [49]. La PCR puede ser un mediador veraz de la asociación entre la periodontitis y resultados adversos del embarazo. El aumento de la PCR puede amplificar la respuesta inflamatoria a través de la activación del complemento, el daño tisular, y la inducción de citoquinas inflamatorias en los monocitos puede mediar la relación entre la periodontitis y los resultados adversos del embarazo. Por otra parte, la enfermedad periodontal y la PCR pueden compartir un factor de riesgo común en el que predisponen a ciertos individuos a una híperrespuesta inflamatoria, cuya relación existente puede ser o no causal. Sharma y colaboradores, en el 2010, citado en Mangalore, realizaron un estudio de cohorte que demuestra que la PCR se encuentra elevada en el grupo de casos de mujeres embarazadas con enfermedad periodontal, en comparación con el grupo control, y la tasa de parto prematuro es mayor en mujeres con enfermedad periodontal [43]; es decir, los niveles de PCR fueron significativamente elevados en embarazadas que presentaban enfermedad periodontal, y se asocian con un mayor riesgo de parto prematuro [50]. También hallaron que el tratamiento periodontal antes de la semana 28 de gestación causó una disminución significativa en los niveles medios de PCR. Ensayos 
aleatorios de tratamiento periodontal han indicado que las infecciones de tipo periodontal pueden bajar significativamente los niveles séricos de PCR [51].

\section{Conclusiones}

Llegar a una conclusión sólida y verdadera es complejo porque hay un manejo de variables y parámetros difíciles de comparar, como puede ser el caso en que una mujer en estado de embarazo y que presente una encía sana probablemente no difiere metabólicamente en comparación con mujeres que no están embarazadas; por otro lado, la respuesta de la encía a los irritantes locales durante el embarazo es muy diferente a la respuesta de aquellas que no se encuentran en este estado. Cabe recordar que existe evidencia sobre el comportamiento celular y bioquímico que puede dar explicación a estos cambios en la cavidad oral durante el embarazo; aun así una madre con adecuados hábitos de higiene oral durante este periodo tendrá menor riesgo de desarrollar enfermedad periodontal y contrarrestar una respuesta gingival fisiológica. Es importante tener en cuenta los mecanismos protectores frente a respuestas inflamatorias como son los linfocitos $\mathrm{T}$ y la proteína $C$ reactiva ( $\mathrm{PCR}$ ), que cumplen un papel fundamental frente a la presencia de la enfermedad periodontal. De igual forma es prioritario evaluar factores socioeconómicos, demográficos y nivel educativo de la madre, que influyen en su higiene oral, y fomentar programas de promoción para el cuidado dental antes, durante y después del embarazo.

\section{Referencias}

[1] Giglio JA, Lanni SM, Laskin DM, Giglio NW. Oral health care for the pregnant patient. Todays FDA. 2011; 23(5): 47-53.

[2] Amar S, Chung KM. Influence of hormonal variation on the periodontium in women. Periodontol 2000. 1994; 6: 79-87.

[3] Maier AW, Orban B. Gingivitis in pregnancy. Oral Surg. 1949; 2: 334-73.

[4] Katz J, Chegini N, Shiverick KT, and Lamont RJ. Localization of P. gingivalis in Preterm delivery Placenta. J Dent Res. 2009; 88: 575.

[5] Cadden KA, Walsh SW. Neutrophils, but not lymphocytes or monocytes, infiltrate maternal systemic vasculature in women with preeclampsia. Hypertens Pregnancy. 2008; 27(4): 396-405.

[6] Otenio CC, Fonseca I, Martins MF, Ribeiro LC, Assis NM, Ferreira AP et al. Expression of IL-1 $\beta$, IL-6, TNF- $\alpha$, and iNOS in pregnant women with periodontal disease. Genet Mol Res. 2012; 11(4): 4468-78.

[7] Heimonen A, Rintamäki H, Furuholm J, Janket SJ, Kaaja $\mathrm{R}$, Meurman JH. Postpartum oral health parameters in women with preterm birth. Acta Odontol Scand. 2008; 66(6): 334-41.

[8] Calabrese N, Calabrese A, Nibali L, Rosati A, Fiengo $\mathrm{S}, \mathrm{Di}$ Renzo GC. Is there any association between periodontitis and preterm low birth weight? J Matern Fetal Neonatal Med. 2010; 23(11): 1288-93.

[9] Piscoya MD, Ximenes RA, Silva GM, Jamelli SR, Coutinho SB. Periodontitis-associated risk factors in pregnant women. Clinics (Sao Paulo). 2012; 67(1): 27-33.

[10] Patcas R, Schmidlin PR, Zimmermann R, Gnoinski W. Dental care in pregnancy. Ten questions and answers. Schweiz Monatsschr Zahnmed. 2012; 122(9): 729-39.

[11] Reis DM, Pitta DR, Ferreira HM, de Jesús MC, de Moraes ME, Soares MG. Health education as a strategy for the promotion of oral health in the pregnancy period. Cien Saude Colet. 2010; 15(1): 269-76.

[12] Mealey BL, Moritz AJ. Hormonal influences: effects of diabetes mellitus and endogenous female sex steroid hormones on the periodontium. Periodontol 2000. 2003; 32: 59-81.

[13] Krejci CB, Bissada NF. Women's health issues and their relationship to periodontitis. J Am Dent Assoc. 2002; 133(3): 323-9.

[14] Sayar F, Sadat MH, Abbaspour S. Effect of Periodontal Disease on Preeclampsia. Iran J Public Health. 2011; 40 (3): 122-7.

[15] Shub A, Swain JR, Newnham JP. Periodontal disease and adverse pregnancy outcomes. J Matern Fetal Neonatal Med. 2006; 19(9): 521-8.

[16] Saini R, Saini S, Saini SR. Periodontitis: A risk for delivery of premature labor and low birth weight infants. J Nat Sci Biol Med. 2011; 2(1): 50-2.

[17] Agueda A, Echeverría A, Manau C. Association between periodontitis in pregnancy and preterm or low birth weight: Review of the literature. Med Oral Patol Oral Cir Bucal. 2008; 13(9): E609-15.

[18] Kinane D. Periodontitis Modified by Systemic Factors. Ann Periodontol. 1999; 4(1): 54-64.

[19] Weidlich P, Cimões R, Pannuti CM, Oppermann RV. Association between periodontal diseases and systemic diseases. Braz Oral Res. 2008; 22 Suppl. 1: 32-43. 
[20] Abraham Inpijn L, Polsacheva OV, Raber Durlacher JE. The significance of endocrine factors and microorganisms in the development of gingivitis in pregnant women. Stomatologiia (Mosk). 1996; 75(3): 15-8.

[21] Moore S, I de M, Wilson RF, Coward PY, Borkowska E, Baylis R, Bewley S, Maxwell DJ, Mulhair L, Ashley FP. Periodontal health of London women during early pregnancy. Br Dent J. 2001; 191(10): 570-3.

[22] Loe H, Silness J. Periodontal disease in pregnancy. I. Prevalence and severity. Acta Odontol Scand. 1963; 21: 533-51.

[23] Silness J, Loe H. Periodontal disease in pregnancy. II. Correlation between oral hygiene and periodontal condition. Acta Odontol Scand. 1964; 22: 121-35.

[24] Ringsdorf WM Jr, Flatland RF, Lindsey KG, Cheraskin E. Clinical tooth mobility and pregnancy. Obstet Gynecol. 1961; 17: 434-8.

[25] Diaz LM, Castellanos JL. Lesions of the oral mucosa and periodontal disease behavior in pregnant patients. Med Oral Patol Oral Cir Bucal. 2004; 9(5): 434-7; 430-3.

[26] Morgan MA, Crall J, Goldenberg RL, Schulkin J. Oral health during pregnancy. J Matern Fetal Neonatal Med. 2009; 22(9): 733-9.

[27] Machado FC, Evangelista CD, Assis DA, Diniz CG, Ribeiro R. Detection and enumeration of periodontopathogenic bacteria in subgingival biofilm of pregnant women. Braz Oral Res. 2012; 26(5): 443-9.

[28] Wandera M, Astrom NA, Okullo I, Tumwine J. Determinants of periodontal health in pregnant women and association with infants' anthropometric status: a prospective cohort study from Eastern Uganda. BMC Pregnancy Childbirth. 2012; 12: 90.

[29] Vogt M, Sallum AW, Cecatti JG, Morais S. Factors associated with the prevalence of periodontal disease in low-risk pregnant women. Reprod Health. 2012; 9: 3.

[30] Organización Mundial de la Salud. Nomen- clatzrre Regulations, Official Records n. ${ }^{\circ}$ 13, Ginebra, 1948; 349-52.

[31] Ramchandani M, Siddiqui M, Kanwar R, Lakha M, Phi L, Giacomelli L et al. Proteomicsignature of periodontal disease in pregnancy: Predictive validity for adverse outcomes. Bioinformation. 2011; 6; 5(7): 300-3.

[32] Holbrook WP, Oskarsdóttir A, Fridjónsson T, Einarsson H, Hauksson A, Geirsson RT. No link between lowgrade periodontal disease and preterm birth: a pilot study in a healthy Caucasian population. Acta Odontol Scand. 2004; 62(3): 177-9.

[33] Mitchell-Lewis D, Engebretson SP, Chen J, Lamster IB, Papapanou PN. Periodontal infections and pre-term birth: early findings from a cohort of young minority women in New York. Eur J Oral Sci. 2001; 109(1): 34-9.
[34] Sadatmansouri S, Sedighpoor, Aghaloo. Effects of periodontal treatment phase I on birth term and birth weight. J Indian Soc Pedod Prev Dent. 2006; 24(1): 23-6.

[35] Ademuyiwa O, Odusoga OL, Adebawo OO, Ugbaja RN. Endogenous antioxidant defenses in plasma and erythrocytes of pregnant women during different trimesters of pregnancy. Acta Obstet Gynecol Scand. 2007; 2: 1-6.

[36] Michalowicz BS, DiAngelis AJ, Novak MJ, Buchanan W, Papapanou PN, Mitchell DA et al. Examining the safety of dental treatment in pregnant women. J Am Dent Assoc. 2008 ; 139(6): 685-95.

[37] Jafarzadeh H, Sanatkhani M, Mohtasham N. Oral pyogenic granuloma: a review. J Oral Sci. 2006; 48(4): 167-75.

[38] LucE,CoulibalyN,DemoersmanJ,BoutignyH,Soueidan A. Dental care during pregnancy. Schweiz Monatsschr Zahnmed. 2012; 122(11): 1047-63.

[39] Effiom OA, Adeyemo WL, Soyele OO. Focal Reactive lesions of the Gingiva: An Analysis of 314 cases at a tertiary Health Institution in Nigeria. Niger Med J. 2011; 52(1): 35-40.

[40] Mannem S, Chava VK. The relationship between maternal periodontitis and preterm low birth weight: A case-control study. Contemp Clin Dent. 2011; 2(2): 88-93.

[41] Sant'Ana AC, Campos MR, Passanezi SC, Rezende ML, Greghi SL, Passanezi E. Periodontal treatment during pregnancy decreases the rate of adverse pregnancy outcome: a controlled clinical trial. J Appl Oral Sci. 2011; 19(2): 130-6.

[42] Shanthi V, Vanka A, Bhambal A, Saxena V, Saxena S, Kumar S. Association of pregnant women periodontal status to preterm and low-birth weight babies: A systematic and evidence-based review. Dent Res J. 2012; 9(4): 368-80.

[43] Sharma A, Ramesh A, Thomas B. Evaluation of plasma C-reactive protein levels in pregnant women with and without periodontal disease: A comparative study. J Indian Soc Periodontol. 2009; 13(3): 145-9.

[44] Pitiphat W, Joshipura KJ, Rich-Edwards JW, Williams PL, Douglass CW, Gillman MW. Periodontitis and Plasma C-Reactive Protein during Pregnancy. J Periodontol. 2006; 77(5): 821-5.

[45] Souccar NM, Chakhtoura M, Ghafari JG, Abdelnoor AM. Porphyromonas gingivalis in dental plaque and serum C-reactive protein levels in pregnancy. J Infect Dev Ctries. 2010; 30; 4(6): 362-6.

[46] Lin D, Smith MA, Champagne C, Elter J, Beck J, Offenbacher S. Porphyromonasgingivalis infection during pregnancy increases maternal tumor necrosis factor alpha, suppresses maternal interleukin-10, and enhances 
fetal growth restriction and resorption in mice. Infect Immun. 2003; 71(9): 5156-62.

[47] Versen-Hoeynck FM, Hubel CA, Gallaher MJ, Gammill S, Powers R. Plasma levels of inflammatory markers neopterin, sialic acid and c-reactive protein in pregnancy and preeclampsia. Am J Hypertens. 2009; 22(6): 687-92.

[48] Shira Davenport E. Preterm low birthweight and the role of oral bacteria. J Oral Microbiol. 2010;21: 2 .
[49] Rawlinson WD, Hall B, Jones CA, Jeffery HE, Arbuckle $\mathrm{SM}, \mathrm{Graf} \mathrm{N}$ et al. Viruses and other infections in stillbirth: what is the evidence and what should we be doing? Pathology. 2008; 40(2): 149-60.

[50] Cudihy D, Lee RV. The pathophysiology of pre-eclampsia: Current clinical concepts. J Obstet Gynaecol. 2009; 29(7): 576-82.

[51] Babalola DA, Omole F. Periodontal Disease and Pregnancy Outcomes. J Pregnancy. 2010; 2010: doi: 10.1155 /2010/293439. 